\title{
Confirmación de la presencia del tiburón cadena, Scyliorhinus retifer (Garman, 1881), en el suroeste del Golfo de México
}

\section{Confirmation of the presence of Scyliorhinus retifer (Garman, 1881) in the southwestern Gulf of Mexico}

\author{
Luis Fernando Del Moral-Flores ${ }^{1 *}$ y Sheila Paleo-Delgado ${ }^{1}$
}

\begin{abstract}
RESUMEN
El tiburón cadena, Scyliorhinus retifer, es una especie demersal ovípara del Atlántico noroccidental, el límite sur de distribución no es preciso. En el Golfo de México la mayoría de sus registros corresponden al área nororiental, con registros esporádicos. Reportamos la presencia de la especie y su morfometría con base en dos especímenes macho maduros, 518 y $527 \mathrm{~mm}$ de longitud total, capturados en la costa suroccidental del Golfo de México.
\end{abstract}

Palabras clave: Chondrichthyes, Carcharhiniformes, Scyliorhinus, Atlántico, México

\section{ABSTRACT}

The chain catshark, Scyliorhinus retifer, is an oviparous demersal species from the northwestern Atlantic. The southern limit of distribution of this species is not clearly established. In the Gulf of Mexico, most of its records correspond to the northeastern area and are sporadic. The presence of the species and its morphometry is reported here based on two mature male specimens, 518 and $527 \mathrm{~mm}$ in total length, captured on the southwestern coast of the Gulf of Mexico.

Keywords: Chondrichthyes, Carcharhiniformes, Scyliorhinus, Atlantic, Mexico

\section{INTRODUCCIÓN}

La familia Scyliorhinidae es conocida como los tiburones pejegato, está representada por 69 especies y 9 géneros (Frike et al. 2018). De ellos, el género Scyliorhinus Blainville, 1816 es el segundo más diverso con 16 especies, y se caracteriza por presentar: dos aletas dorsales, el origen de la primera dorsal ligeramente anterior a la parte posterior de base de la aleta pélvica; aleta pectoral pequeña con esquinas redondeadas; aleta anal pequeña, con inicio anterior al origen de la segunda aleta dorsal;

1 Laboratorio de Zoología, Facultad de Estudios Superiores Iztacala, Universidad Nacional Autónoma de México (UNAM).delmoralfer@comunidad.unam.mx*,sheila.paleodel@gmail.com 
hocico corto con punta redondeada; cuarta y quinta hendiduras branquiales por encima de la base de la aleta pectoral; fosas nasales cercanas a la boca; surco labial de la mandíbula inferior pequeño y ausencia del surco labial superior; crestas supraorbitales presentes, además de un patón de coloración representado por manchas a manera de silla de montar, manchas oscuras o claras (Nakaya, 1975; Springer, 1979; Compagno, 1984).

En el litoral atlántico de México, se han registrado tres especies del género Scyliorhinus: S. hesperius Springer, 1966, S. meadi Springer, 1966 y S. retifer (Garman, 1881) (Del Moral-Flores et al. 2015). Esta última es conocida como tiburón cadena, es una especie demersal ovípara, cuya alimentación incluye poliquetos, calamares, peces óseos y crustáceos (Castro et al. 1988). Es una especie endémica del Atlántico noroccidental, su límite norteño se restringe a los bancos de Nueva Escocia, Canadá, mientras que el límite sureño de distribución aún no es preciso, aunque se ha señalado a las aguas de Nicaragua (Gilhen et al. 2003; Castro, 2011).

En el Golfo de México la mayoría de sus registros corresponden al área nororiental (Castro, 2011). Hasta el momento solo existen tres registros esporádicos, con base en ejemplares depositados en colecciones, en la región suroeste de este mar (Del Moral-Flores et al. 2015). Como objetivo queremos presentar la evidencia de un registro adicional y la morfometría completa de dos ejemplares de $S$. retifer capturados en la plataforma continental de Veracruz, México.

\section{MATERIALES Y MÉTODOS}

En el mes de diciembre de 2017, se capturaron incidentalmente dos ejemplares de la especie Scyliorhinus retifer, dentro de la pesca tiburonera realizada por los pescadores ribereños de la localidad de Antón Lizardo, municipio de Alvarado, Veracruz, México. El ejemplar fue capturado por medio de palangre de fondo sobre una profundidad cercana a los $20 \mathrm{~m}$, en la plataforma continental de Veracruz, en un área cercana a la Isla Santiaguillo (1908' 19.4"' N, 95 47'43.1'” W), Parque Nacional Sistema Arrecifal Veracruzano (Fig. 1).

Los ejemplares fueron donados y depositados bajo los números de catálogo CIFI 1409 y CIFI 1410 en la Colección Ictiológica de la Facultad de Estudios Superiores Iztacala (CIFI), Universidad Nacional Autónoma de México. Se corroboró su identidad mediante las claves de Springer (1979) y Castro (2011). Se les determinó el sexo, así como la madurez mediante la calcificación del mixopterigio (Colonello et al. 2011). Se obtuvieron en total 75 medidas morfométricas de acuerdo con Compagno (2001). 


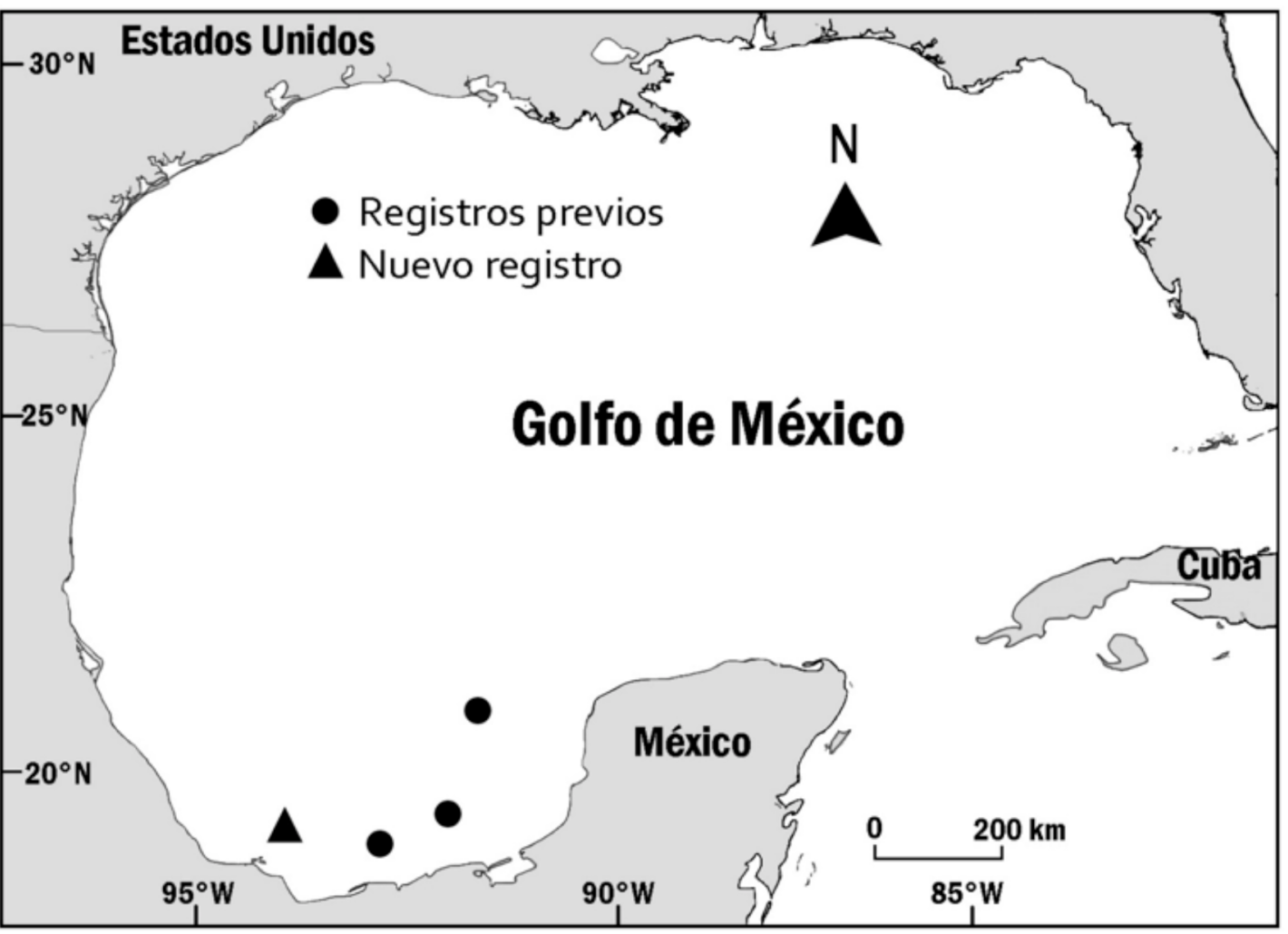

Fig. 1. Registros previos de Scyliorhinus retifer en el suroeste del Golfo de México y nueva localidad, en México

Fig. 1. Previous records of Scyliorhinus retifer in the Southwestern Gulf of Mexico and new location in Mexico

\section{RESULTADOS Y DISCUSIÓN}

Los dos ejemplares capturados fueron identificados como $S$. retifer, especímenes macho maduros $(518 \mathrm{y}$ $527 \mathrm{~mm}$ de longitud total), y fueron reconocidos por la siguiente combinación de características: cuerpo alargado y hocico triangular, con un distintivo patrón de coloración a manera de cadena color marrón o negro en todo el cuerpo; ojos grandes y ovalados; aletas dorsales lobuladas y cercanas a la aleta caudal; aleta caudal asimétrica y con muescas; pliegues nasales no alcanzan el borde del labio superior; dientes triangulares, con una gran cúspide central bordeada por una cúspide pequeña a cada lado; presencia de crestas supraoculares (Fig. 2). Las medidas morfométricas y proporciones corporales de los dos ejemplares 
(Cuadro 1) corresponden a los valores señalados para otras poblaciones a lo largo de su distribución (Gilhen et al. 2003; Castro, 2011).

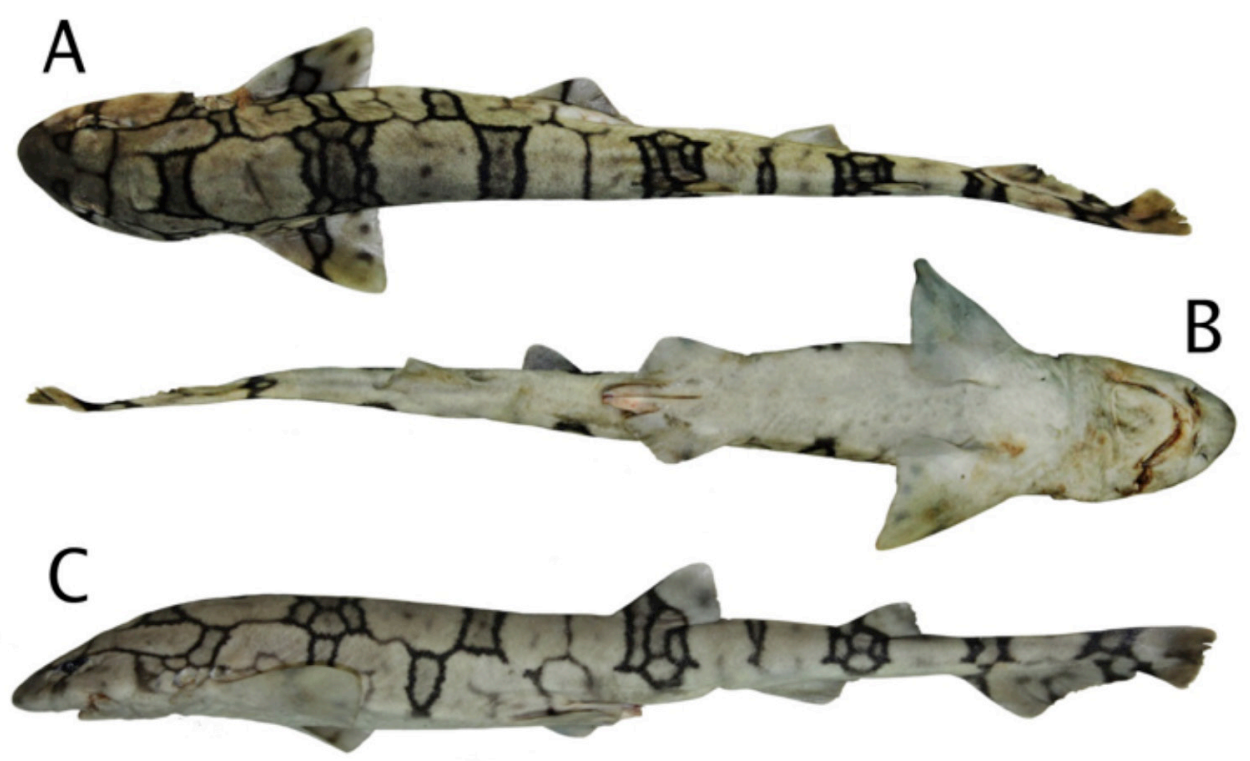

Fig. 2. Vista dorsal (A), ventral (B) y lateral (C) de un ejemplar de tiburón cadena, Scyliorhinus retifer (macho, $527 \mathrm{~mm}$ de LT) capturado en el suroeste del Golfo de México

Fig. 2. Dorsal (A), ventral (B) and lateral (C) view of a chain catshark, Scyliorhinus retifer (male, $527 \mathrm{~mm}$ TL) collected in the Southwestern Gulf of Mexico

Cuadro 1. Medidas morfométricas $(\mathrm{mm})$ de los organismos de Sclyorhinus retifer capturados en el Suroeste del Golfo de México

Table 1. Morphometric measurements ( $\mathrm{mm}$ ) of the Sclyorhinus retifer organisms captured in the Southwestern Gulf of Mexico

\begin{tabular}{lcc}
\hline Morfometría $(\mathbf{m m})$ & CIFI 1409 & CIFI 1410 \\
\hline Sexo & Macho & Macho \\
\hline Longitud total & 527 & 518 \\
Longitud precaudal & 420 & 405 \\
Longitud predorsal, segunda dorsal & 360 & 355 \\
Longitud predorsal, primera dorsal & 267 & 267
\end{tabular}




\begin{tabular}{|c|c|c|}
\hline Morfometría (mm) & CIFI 1409 & CIFI 1410 \\
\hline Sexo & Macho & Macho \\
\hline Longitud precloacal & 235 & 219 \\
\hline Longitud prepélvica & 220 & 215 \\
\hline Longitud prepectoral & 119 & 90.7 \\
\hline Longitud cefálica & 80.1 & 76 \\
\hline Longitud hocico-primera hendidura branquial & 104.8 & 107 \\
\hline Longitud preespiracular & 57.07 & 53 \\
\hline Longitud preorbital & 36.89 & 33.9 \\
\hline Longitud prenasal externa & 23.17 & 21.8 \\
\hline Longitud preoral & 27.18 & 26.7 \\
\hline Longitud prenasal interna & 22.8 & 20.8 \\
\hline Espacio internasal-labial & 28.2 & 20.6 \\
\hline Ancho de boca & 37.29 & 33.2 \\
\hline Surco labial & 5.4 & 4.2 \\
\hline Espacio internasal & 25.42 & 24 \\
\hline Interorbital & 31.9 & 31.3 \\
\hline Longitud del ojo & 14.5 & 14.3 \\
\hline Altura del ojo & 3.7 & 2.4 \\
\hline Ancho máximo del espiráculo & 1.7 & 2.3 \\
\hline Altura primer hendidura branquial & 9.6 & 9 \\
\hline Altura quinta hendidura branquial & 5.6 & 6 \\
\hline Longitud interdorsal & 57.1 & 46 \\
\hline Longitud dorsal-caudal & 26.1 & 23.4 \\
\hline Distancia pélvico-pectoral & 92.9 & 90.6 \\
\hline Distancia pélvico-caudal & 146.1 & 141.1 \\
\hline Longitud extensión de la primera dorsal & 47.9 & 46.6 \\
\hline Margen anterior de la primer dorsal & 51.2 & 48.8 \\
\hline Base de la primer dorsal & 32.4 & 31.1 \\
\hline Altura de la primer dorsal & 28.4 & 26.2 \\
\hline Margen interno de primer dorsal & 14.2 & 15.3 \\
\hline Margen posterior de la primer dorsal & 27.2 & 25.1 \\
\hline Longitud extensión de la segunda dorsal & 39.6 & 38.7 \\
\hline Margen anterior segunda dorsal & 36.8 & 36.1 \\
\hline Base de la segunda dorsal & 28.2 & 27.4 \\
\hline Altura de la segunda dorsal & 15.5 & 15.4 \\
\hline
\end{tabular}




\begin{tabular}{|c|c|c|}
\hline Morfometría (mm) & CIFI 1409 & CIFI 1410 \\
\hline Sexo & Macho & Macho \\
\hline Margen interno de la segunda dorsal & 13.7 & 12.1 \\
\hline Margen posterior de la segunda dorsal & 16.4 & 14.6 \\
\hline Margen anterior de la pectoral & 71.6 & 67.3 \\
\hline Margen interno de la pectoral & 31.2 & 32.2 \\
\hline Base de la pectoral & 30.9 & 31.8 \\
\hline Margen posterior de la pectoral & 50.6 & 49.7 \\
\hline Longitud extensión de la aleta pélvica & 63.4 & 61.8 \\
\hline Altura de la pélvica & 11.6 & 12.7 \\
\hline Margen interno de la pélvica & 24.1 & 24.1 \\
\hline Longitud del lóbulo caudal superior & 111 & 109.5 \\
\hline Longitud del lóbulo caudal inferior & 24.7 & 42.4 \\
\hline Distancia horquilla caudal al ápice del lóbulo superior & 40.9 & 35 \\
\hline Distancia horquilla caudal al ápice del lóbulo inferior & 54.5 & 51.6 \\
\hline $\begin{array}{l}\text { Longitud perpendicular de la horquilla caudal al lóbulo } \\
\text { superior }\end{array}$ & 18.3 & 17.8 \\
\hline $\begin{array}{l}\text { Longitud perpendicular de la horquilla caudal al lóbulo } \\
\text { inferior }\end{array}$ & 73.3 & 73.3 \\
\hline Anchura cefálica en el nivel de las fosas nasales & 37.7 & 33.1 \\
\hline $\begin{array}{l}\text { Anchura cefálica en el nivel del margen anterior de la } \\
\text { boca }\end{array}$ & 61.2 & 33 \\
\hline $\begin{array}{l}\text { Anchura cefálica en el nivel de la quinta hendidura bran- } \\
\text { quial }\end{array}$ & 58.6 & 53.8 \\
\hline Ancho del tronco & 50.3 & 47 \\
\hline Ancho del abdomen & 21.3 & 20 \\
\hline $\begin{array}{l}\text { Ancho del cuerpo en el nivel de la inserción de la aleta } \\
\text { pélvica }\end{array}$ & 37.1 & 29.9 \\
\hline Ancho del pedúnculo caudal & 9.6 & 9.7 \\
\hline $\begin{array}{l}\text { Altura cefálica en el nivel de la quinta hendidura bran- } \\
\text { quial }\end{array}$ & 50 & 44.8 \\
\hline Altura del tronco & 48.9 & 52.5 \\
\hline Altura del abdomen & 30.3 & 34.3 \\
\hline $\begin{array}{l}\text { Altura corporal en el nivel de la inserción de la aleta } \\
\text { pélvica }\end{array}$ & 35.3 & 31.5 \\
\hline Altura del pedúnculo caudal & 15 & 13.3 \\
\hline Longitud exterior del mixopterigio & 24.1 & 20.9 \\
\hline
\end{tabular}




\begin{tabular}{lcc}
\hline Morfometría (mm) & CIFI 1409 & CIFI 1410 \\
\hline Sexo & Macho & Macho \\
\hline Longitud interior de mixopterigio & 31.3 & 27 \\
Ancho de la base de mixopterigios & 6.2 & 4.4 \\
Longitud extensión de la aleta anal & 49.3 & 48.7 \\
Altura de la aleta anal & 37.4 & 35.9 \\
Base de la aleta anal & 38.5 & 35.2 \\
Altura de la aleta anal & 20.7 & 19.1 \\
Margen interno de la aleta anal & 15.3 & 17.8 \\
Margen posterior de la aleta anal & 26.4 & 26.7 \\
Distancia preanal & 327 & 317 \\
\hline
\end{tabular}

Aunque esta especie ha sido registrada en el Atlántico central occidental, ha sido de manera esporádica e irregular, ya que los adultos prefieren terrenos irregulares que les proporciona refugio (Kyne et al. 2012). La presencia de organismos maduros puede indicar la posibilidad de zonas propias para su reproducción en áreas colindantes del Golfo de México. Se ha sugerido que $S$. retifer deposita sus huevos en hábitats estructurados que suelen servir como áreas de crianza después de la eclosión (Able \& Flescher, 1991). Al ser el sustrato un factor determinante para la distribución de ciertas especies de condrictios ovíparas (Ellis et al. 2004).

Las condiciones oceanográficas presentes en el Sistema Arrecifal Veracruzano, cerca del área de captura de este registro, son complejas: plataforma continental en forma de arco, estrecha (aproximadamente de $30 \mathrm{~km}$ ), con una profundidad promedio de
$70 \mathrm{~m}$; topografía heterogénea por la presencia de bancos de arena, zonas arrecifales e islas que forman entre sí una serie de canales (Salas-Pérez \& Granados-Barba, 2008). Existe, además aporte terrígeno por la descarga de los principales ríos de La Antigua, Jamapa y Papaloapan (Krutak, 1997). Con respecto a los procesos oceánico-atmosféricos, como las corrientes marinas, varían a lo largo del año, incluso con una marea de tipo diurna. Los frentes atmosféricos, conocidos localmente como "nortes" producen importantes mezclas en la columna de agua, y conducen a conexiones entre los elementos pelágicos y bentónicos (Gallucci \& Netto, 2004; Salas-Pérez \& Granados-Barba, 2008). Este conjunto de condiciones puede servir como zonas de refugio, con fines reproductivos o ambas.

Los recientes registros de condrictios en el Atlántico central occidental, propios de zonas templadas, 
como es el caso de Mitsukurina owstoni (Parsons et al. 2002; Driggers III et al. 2014), Zameus squamulosus (Villalobos et al. 2016) y S. hesperius en Guatemala (Hacohen-Domené et al. 2016), nos pueden indicar que al igual que $S$. retifer, los intervalos de distribución pueden ser mayores al considerar la continuidad térmica y la baja temperatura, que ocurre a mayor profundidad en la zona tropical. Esta relación batimétrica puede explicar las discontinuidades y las variaciones geográficas de $S$. retifer a lo largo de su distribución. En latitudes norteñas es abundante cerca de la plataforma continental en aguas poco profundas, cercana a los $73 \mathrm{~m}$, aunque su intervalo batimétrico se encuentra debajo de $450 \mathrm{~m}$ en la pendiente continental (Compagno et al. 2005). En cambio, en latitudes sureñas, sus registros son ocasionales y tienden a corresponder a aguas más profundas de las laderas continentales, entre los $550 \mathrm{~m} \mathrm{a}$ $754 \mathrm{~m}$ de profundidad (Springer \& Sadowsky, 1970; Able \& Flescher, 1991; Compagno et al. 2005).

\section{AGRADECIMIENTOS}

Agradecemos la cooperación de los pescadores locales, en especial a Leonel Sánchez Ramos, por su ayuda en la fase de muestreo y la donación de los ejemplares del presente estudio. Asimismo, a los revisores anónimos por sus comentarios que ayudaron a mejorar el manuscrito.

\section{BIBLIOGRAFÍA}

Able, K. W. \& Flescher, D. (1991). Distribution and habitat of chain dogfish, Scyliorhinus retifer, in the Mid-Atlantic Bight. Copeia, 1991(1), 231-234.

Castro, J. I. (2011). The sharks of North America. New York, EE. UU.: Oxford University Press.

Castro, J., Overstrom, N. \& Bubucis, P. (1988). The Reproductive Biology of the Chain Dogfish, Scyliorhinus retifer. Copeia, 1988(3), 740-746.

Colonello, J. H., Christiansen, H. E. \& Macchi, G. J. (2011). Escala de madurez sexual para peces cartilaginosos de la Plataforma Continental Argentina. En O. C. Wöhler, P. Cedrola \& M. B. Cousseau (Ed.), Contribuciones sobre la biología, pesca y comercialización de tiburones en la Argentina. Aportes para la elaboración del Plan de Acción Nacional (pp. 115-128). Buenos Aires, Argentina: Consejo Federal Pesquero.

Compagno, L. J. V. (1984). Vol. 4. Sharks of the world: An annotated and illustrated catalogue of shark species known to date. Part 2. Carcharhiniformes. Rome, Italy: FAO.

Compagno, L. J. V. (2001). Sharks of the world. An annotated and illustrated catalogue of shark species known to date. Volume 2. Bullhead, mackerel and carpet sharks (Heterodontiformes, Lamniformes and Orectolobiformes). Rome, Italy: FAO.

Compagno, L. J. V., Dando, M. \& Fowler, S. L. (2005). Sharks of the World. Princeton, EE. UU.: Princeton University Press. 
Del Moral-Flores, L. F., Morrone, J. J., Alcocer-Durand, J., Espinosa-Pérez, H. \& Pérez-Ponce De León, G. (2015). Lista patrón de los tiburones, rayas y quimeras (Chondrichthyes, Elasmobranchii, Holocephali) de México. Arx. Misc. Zool., 13, 47-163.

Driggers III, W. B., Davis, K. S., Moore, C. \& Carlson, J. K. (2014). New record of a globin shark Mitsukurina owstoni (Lamniformes: Mitsukurinidae) in the western North Atlantic Ocean. Mar. Biodivers. Rec., 7, e96. https://doi. org/10.1017/S1755267214000876Pu

Ellis, J. R., Cruz-Martínez, A., Rackham, B. D. \& Rogers, S. I. (2004). The distribution of Chondrichthyan fishes around the Bristis Isles and Implications for conservation. J. Northw. Atl. Fish. Sci., 35, 195-213.

Fricke, R., Eschmeyer, W. N. \& Fong, J. D. (2018). Species by family/subfamily. Recuperado en octubre 3, 2018. disponible en http://researcharchive.calacademy.org/research/ichthyology/cata$\log$ /SpeciesByFamily.asp).

Gallucci, F. \& Netto, S. A. (2004). Effects of the passage of cold fronts over a coastal site: an ecosystem approach. Mar. Ecol. Progr. Ser., 281, 79-92. https:// doi.org/ 10.3354/meps281079

Gilhen, J., Coad, B. W. \& Hebda, A. (2003). The chain dogfish, Scyliorhinus retifer (Garman, 1881), new to the Canadian Atlantic ichthyofauna. Can. Field Nat., 117(3), 475-477.

Hacohen-Domené, A., Polanco-Vázquez, F. \& Graham, R. T. (2016). First report of the whitesaddled catshark Scyliorhinus hesperius (Springer 1966) in Guatemala's Caribbean Sea. Mar. Biodivers. Rec., 9, 101. https://doi.org/10.1186/ s41200-016-0103-9
Krutak, P. R. (1997). Petrography and provenance of siliciclastic sediments, Veracruz-Antón Lizado Reefs, México. Ocean Polar Res., 19(3), 231-243.

Kyne, P. M., Carlson, J. K., Ebert, D. A., Fordham, S. V., Bizzarro, J. J., Graham, R. T., Kulka, D. W., Tewes, E. E., Harrison, L. R. \& Dulvy, N. K. (2012). The Conservation Status of North American, Central American, and Caribbean Chondrichthyans. Vancouver, Canada: IUCN Species Survival Commission Shark Specialist Group.

Nakaya, K. (1975). Taxonomy, comparative anatomy and phylogeny of Japanese catsharks, Scyliorhinidae. Mem. Fac. Fish. Hokkaido Univ., 23(1), 1-94

Parsons, G. R., Ingram Jr., G. W. \& Haward, R. (2002). First record of the globin shark Mitsukurina owstoni, Jordan (Family Mitsukurinidae) in the Gulf of Mexico. Southeast. Nat., 1(2), 189-192.

Salas-Pérez, J. J. \& Granados-Barba, A. (2008). Oceanographic characterization of the Veracruz reefs system. Atmósfera, 21(3), 281-301.

Springer, S. (1979). A revision of the catsharks, Family Scyliorhinidae. NOAA Tech. Rep. NMFS, Circ., 422, 1-97.

Springer, S. \& Sadowsky, V. (1970). Subspecies of the western Atlantic catshark, Scyliorhinus retifer. Proc. Biol. Soc. Wash., 83, 83-98.

Villalobos, E., Martínez, A., Lambarri, C. \& Espinosa, H. (2016). New record of Zameus squamulosus (Chondrichthyes: Squaliformes: Somniosidae) in the Southern Gulf of México. Mar. Biodivers. Rec., 9, 1-6. https://doi. org/10.1186/s41200-016-0047-0 
